\title{
Exploring the Biological and Mechanical Properties of Abdominal Aortic Aneurysms Using USPIO MRI and Peak Tissue Stress: A Combined Clinical and Finite Element Study
}

\author{
Noel Conlisk ${ }^{1,2,3}$ (D) Rachael O. Forsythe ${ }^{1,2,4}$. $^{\text {Lyam Hollis }}{ }^{1} \cdot$ Barry J. Doyle $^{1,5,6}$. \\ Olivia M.B. McBride ${ }^{1,2,4}$ • Jennifer M.J. Robson ${ }^{1,2}$ • Chengjia Wang ${ }^{1,4}$. \\ Calum D. Gray ${ }^{4}$. Scott I.K. Semple ${ }^{1,4}$. Tom MacGillivray ${ }^{1,7}$ • Edwin J.R. van Beek ${ }^{1,4}$. \\ David E. Newby ${ }^{1,4}$ - Peter R. Hoskins ${ }^{1,3}$
}

Received: 11 January 2017 / Accepted: 4 August 2017 /Published online: 14 August 2017

(C) The Author(s) 2017. This article is an open access publication

\begin{abstract}
Inflammation detected through the uptake of ultrasmall superparamagnetic particles of iron oxide (USPIO) on magnetic resonance imaging (MRI) and finite element (FE) modelling of tissue stress both hold potential in the assessment of abdominal aortic aneurysm (AAA) rupture risk. This study aimed to examine the spatial relationship between these two biomarkers. Patients $(n=50)>40$ years with AAA maximum diameters $>=40 \mathrm{~mm}$ underwent USPIO-enhanced MRI and computed tomography angiogram (CTA). USPIO uptake was compared with wall stress predictions from CTA-based patient-specific FE models of each aneurysm. Elevated stress
\end{abstract}

Associate Editor Emanuele Barbato oversaw the review of this article

Electronic supplementary material The online version of this article (doi:10.1007/s12265-017-9766-9) contains supplementary material, which is available to authorized users.

Noel Conlisk

noel.conlisk@ed.ac.uk

1 Centre for Cardiovascular Science, The University of Edinburgh, Edinburgh, UK

2 School of Clinical Sciences, The University of Edinburgh, Edinburgh, UK

3 Institute for Bioengineering, The University of Edinburgh, Faraday Building, The King's Buildings, Mayfield Road, Edinburgh EH9 3JL, UK

4 Clinical Research Imaging Centre, The University of Edinburgh, Edinburgh, UK

5 Vascular Engineering Laboratory, Harry Perkins Institute of Medical Research, Perth, Australia

6 School of Mechanical and Chemical Engineering, The University of Western Australia, Perth, Australia

7 Centre for Clinical Brain Sciences, The University of Edinburgh, Edinburgh, UK was commonly observed in areas vulnerable to rupture (e.g. posterior wall and shoulder). Only $16 \%$ of aneurysms exhibited co-localisation of elevated stress and mural USPIO enhancement. Globally, no correlation was observed between stress and other measures of USPIO uptake (i.e. mean or peak). It is suggested that cellular inflammation and stress may represent different but complimentary aspects of AAA disease progression.

Keywords Abdominal aortic aneurysms $\cdot$ Finite element analysis $\cdot$ USPIO uptake $\cdot$ MRI $\cdot$ Patient-specific modelling

\section{Introduction}

Each year, over 10,000 deaths in the UK are attributed to rupture of abdominal aortic aneurysms (AAAs) [1]. It is thought that AAA rupture occurs when wall stress exceeds wall strength, and this is influenced by a number of biological and mechanical factors [2]. However, the exact mechanisms of AAA rupture are unknown. Various pathobiological processes contributing to AAA development and disease progression have been identified, including infiltration of inflammatory cells such as macrophages, proteolytic degradation of the extracellular matrix (ECM), and neovascularisation. All of these biological processes lead to changes in the mechanical properties of the artery wall including loss of elastin and deposition of collagen that compromises the strength and elasticity of the vessel $[3,4]$.

It is clear that growth and ultimately rupture of AAAs over time occur as a result of complex mechano-biological interactions within the diseased arterial wall [5]. However, it is unclear to what extent these biological and mechanical changes may co-exist and, indeed, contribute to the propagation of each other. Novel imaging studies have been undertaken in 
an attempt to understand the biological activity of AAA disease, such as the application of ${ }^{18} \mathrm{~F}$-fluoride to detect necrotic inflammation [6] and microcalcification [7] or the application of ultrasmall superparamagnetic particles of iron oxide (USPIO) to detect inflammation $[8,9]$. Also, we have previously demonstrated that USPIO can identify areas of mural AAA inflammation, which is associated with more rapid aneurysm expansion [8].

Biomechanical studies can assess properties of AAA, such as wall stress and strength [10], and it has been shown that computational models based on the finite element (FE) method can identify rapidly expanding AAAs [11], assess rupture risk [12], and in some instances may even predict the location of rupture $[13,14]$.

It has been suggested that focal areas of inflammation may be co-located with focal areas of increased mechanical stress [15]. The aim of this study was to explore the spatial relationship between areas of mural cellular inflammation measured by USPIO uptake on MRI and regions of high tissue stress determined through patient-specific FE modelling, for a group of 50 patients under surveillance for AAA disease.

\section{Methods}

\section{Study Design and Setting}

This is a sub-study of the MRI in AAA to predict Rupture or Surgery (MA $\left.{ }^{3} \mathrm{RS}\right)$ study (http://www.isrctn.com/ ISRCTN76413758): a large multi-centre prospective observational cohort study aiming to determine the added value of USPIO-enhanced MRI in predicting AAA rupture or surgery in 342 patients with AAA under routine clinical surveillance. The MA ${ }^{3} R S$ study protocol has been described in full elsewhere [16], and the study is in the follow-up stages, due to report in 2017. In brief, the $\mathrm{MA}^{3} \mathrm{RS}$ study cohort underwent USPIO MRI and CTA between November 2012 and December 2014 at the University of Edinburgh, Scotland. Patients were eligible for inclusion if they had an AAA $\geq 40 \mathrm{~mm}$ as measured on an ultrasound scan, if they were over 40 years of age, and with no contraindications to USPIO MRI or CTA. Patients were excluded if the suspected aetiology of the aneurysm was inflammatory. An outline of the patient selection criteria is given in Fig. 1a.

For the present sub-study, we selected and analysed scans of consecutive patients recruited into the $M A^{3} \mathrm{RS}$ study using the process outlined in Fig. 1b. During this phase, 14 of the available consecutive scans were unable to be processed due to factors such as poor contrast on the CT scan $(n=6)$, segmentation difficulties $(n=4)$, and a lack of corresponding MRI-USPIO data $(n=4)$. These patients were therefore excluded from the final analysis. As per the selection algorithm (Fig. 1b), if a patient became ineligible, reconstruction and analysis moved on to the next consecutive patient until the predetermined target sample size of 50 eligible patients was reached. During the selection and reconstruction phase of the study, we were blinded to all demographic data, clinical outcomes, and USPIO groupings for each patient.

\section{USPIO Magnetic Resonance Imaging and Image Analysis}

The USPIO MRI and image analysis techniques used in the study have previously been described [16]. Briefly, participants underwent T2 and T2*-weighted MRI scanning at $3 \mathrm{~T}$ (Siemens Magnetom Verio, Erlangen, Germany) both before and 24-36 $\mathrm{h}$ after administration of a weight-adjusted dose of USPIO. Both datasets (pre- and post-USPIO) were then registered and analysed, using bespoke software. USPIO causes a rapid decline in $\mathrm{T} 2 *$, which is the constant for the decay of MRI signal intensity over time, and as such, changes in T2* can be used to assess USPIO accumulation in the AAA [8]. Colour maps representing the percentage change in $\mathrm{T} 2 *$ $(\% \Delta \mathrm{T} 2 *)$ were generated using custom written scripts (MATLAB, The MathWorks Inc., Natick, MA). In order to minimise the effect of artefact, a threshold of $\geq 71 \% \Delta \mathrm{T} 2 *$ was used to identify areas of signal change attributable to true USPIO uptake, based on previous reproducibility data from our group [16].

Colour maps were classified according to predefined criteria [8], into USPIO-negative (no mural USPIO uptake) or USPIO-positive (area(s) of significant mural USPIO enhancement indicative of macrophage-driven inflammation). Significant mural USPIO enhancement was defined as 10 or more contiguous USPIO-positive voxels adjacent to the aneurysm wall. Periluminal USPIO uptake is not considered to represent true inflammation and likely represents passive trapping of USPIOs in periluminal thrombus [17] although mural and periluminal uptake can be difficult to distinguish from posterior wall uptake due to their close proximity.

\section{Computed Tomography Three-Dimensional Reconstruction and Meshing}

A standard high-resolution contrast-enhanced CTA was performed with a slice thickness of $1.0 \mathrm{~mm}$ and pixel size of $0.625 \mathrm{~mm}$ (Aquilion One, Toshiba Medical Systems Ltd., UK). These images were used to create patient-specific models of each aneurysm. Segmentation and 3D reconstruction were performed using commercial software (VASCOPS $\mathrm{GmbH}$, Sweden). Early studies of AAA often assumed a uniform wall thickness [18]; however, variable wall thickness has been shown to highly influence the predicted results [12, 19-22]. Therefore, this package employs a specialist algorithm to calculate a more physiological aneurysm wall thickness distribution, which varies between 1.5 and $1.13 \mathrm{~mm}$ at the thrombus-free and covered sites, respectively [19]. Finite 
a

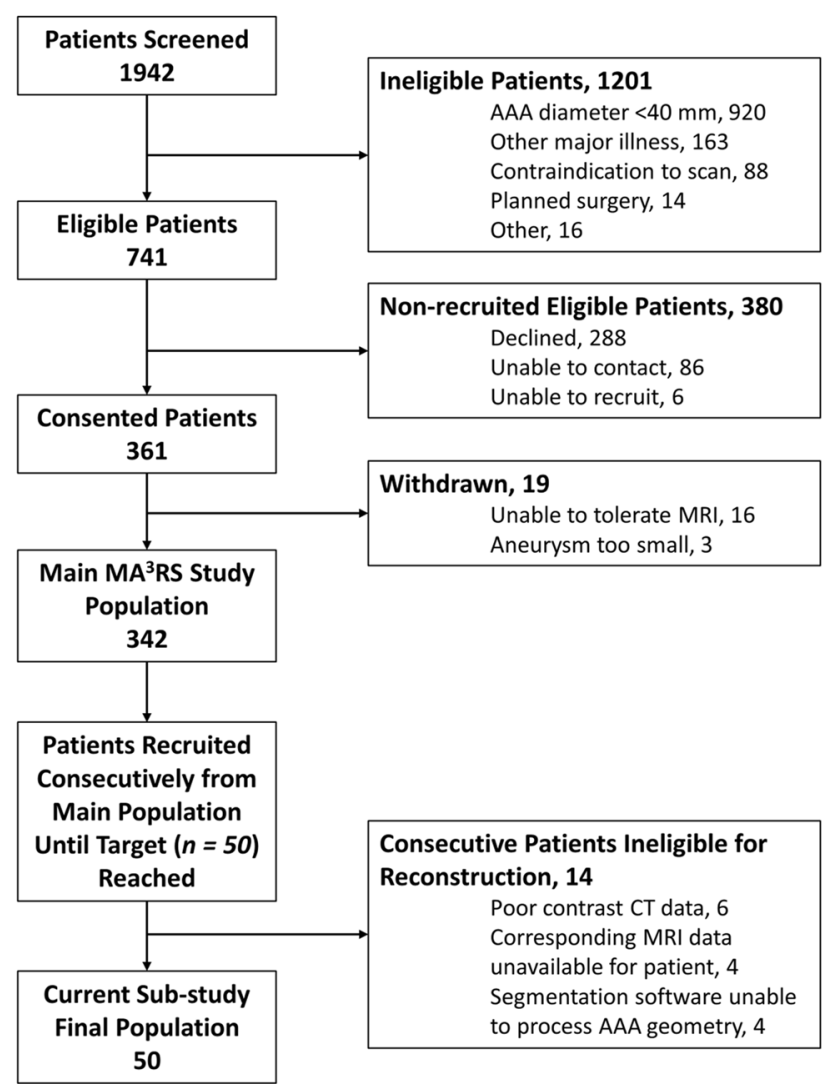

b

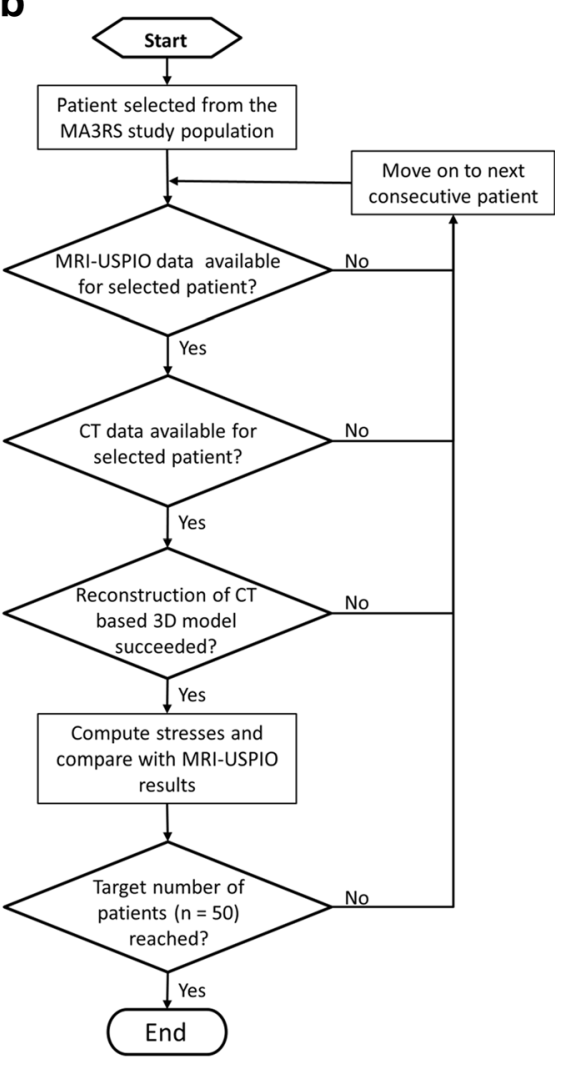

Fig. 1 Flowcharts detailing (a) patient inclusion/exclusion criteria and (b) the patient selection algorithm

element (FE) meshes were then created from the 3D aneurysm geometry using the A4 clinical research software (VASCOPS). After suitable refinement, each AAA volume mesh typically consisted of $>160,000(\mathrm{C} 3 \mathrm{D} 8 \mathrm{H})$ elements. These meshes were then exported to Abaqus 6.10-1 (Dassault Systemes, Simulia, Providence, RI, USA) for analysis. Both the aortic wall and thrombus regions were modelled as hyperelastic, homogeneous, incompressible, and isotropic materials, using well-established constitutive models $[23,24]$ with material constants based on population data. Loading representative of peak systolic blood pressure was applied as an outward-facing uniformly distributed pressure load acting on the luminal surface of the aneurysm. To remove any variability due to loading and to allow for comparison across patient cases, a peak systolic blood pressure of $120 \mathrm{mmHg}(0.016 \mathrm{MPa})$ was chosen, as in many previous studies $[25,26]$. In the present study, the effect of wall shear stress due to blood flow was not considered due to its negligible magnitude. Residual stresses in the aortic wall itself and the interaction of the aorta with the surrounding structures of the body (e.g. organs and spine) were also not considered. However, displacements at the distal and proximal most regions of each aneurysm were restrained, in all degrees of freedom, to model attachment of the AAA to the rest of the aorta.
For efficiency, a custom script was developed in Python (Python Software Foundation, Python Language Reference, version 2.7, available at http://www.python.org) to automate the definition of the model parameters and batch process all 50 patients. All simulations were computed on a Dell Precision T7600 workstation with 16 cores and 64 GB of RAM. Contour plots of von Mises stress were output for all aneurysms, and their locations were manually aligned to USPIO uptake colour maps. Maximum AAA diameter, as measured orthogonal to the AAA centreline, was also extracted from each $\mathrm{CT}$ reconstruction.

\section{Comparison of Two-Dimensional Contour Plots and USPIO Uptake on Colour Maps}

In order to compare the spatial relationship between elevated stress and areas of inflammation represented by USPIO uptake, the two-dimensional (2D) contour maps of von Mises stress were manually co-aligned with the USPIO colour maps. The MRI slice with the largest area of USPIO uptake (i.e. most diseased segment) was chosen for analysis, ensuring that the corresponding cross-sectional slice was analysed from the 2D contour map (Fig. 2a). Regions of elevated stress and areas of mural USPIO enhancement were examined for visual overlap on the chosen slice. 
Fig. 2 (a) The slice with the largest mural USPIO

enhancement (most diseased segment) is selected from MRI.

The corresponding slice is then extracted from the CT-based FE model using relevant anatomical landmarks (e.g. Z-distance from the iliac bifurcation). The contours are then visually compared to determine if co-location occurs. Examples of aneurysms with no co-location, some periluminal colocation (which does not represent inflammation), and true colocation of elevated stress with significant mural USPIO enhancement can be seen in panels (b), (c), and (d), respectively. Note: black arrows point to approximate regions of co-location a

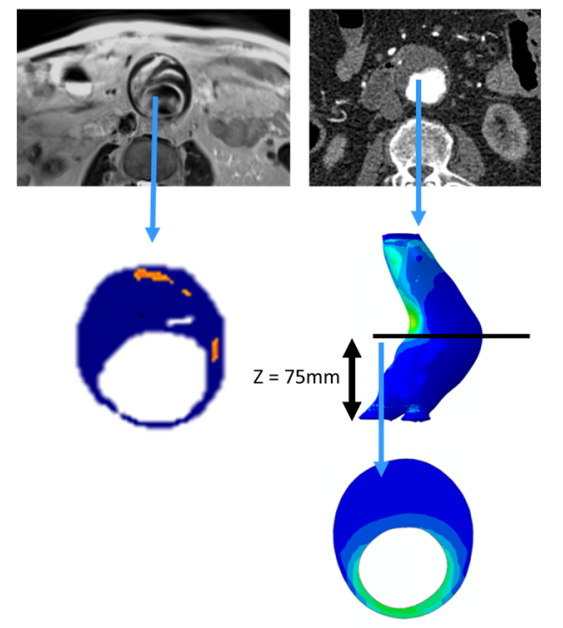

C
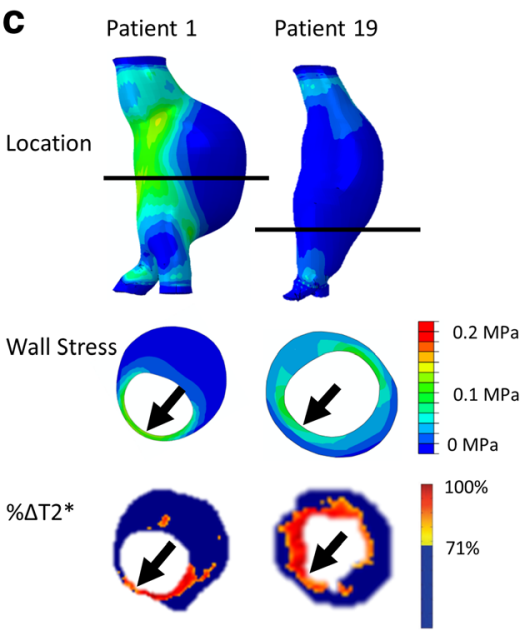

b

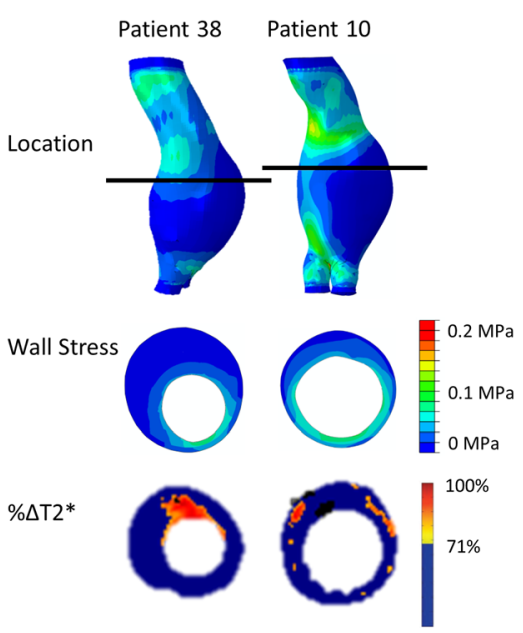

d Patient 12 Patient 35

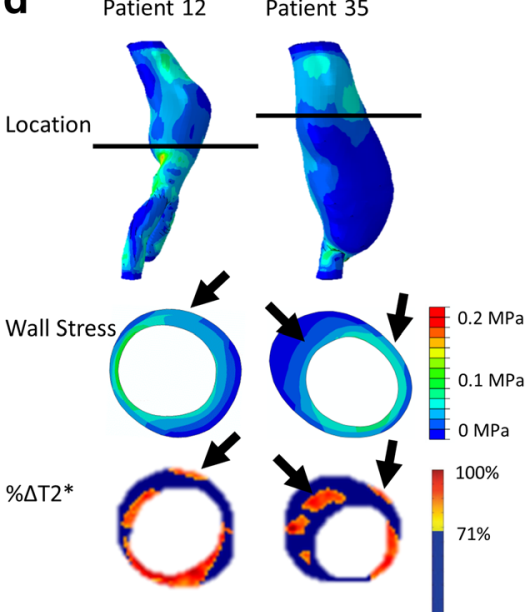

\section{Global Comparisons: Whole AAA Analysis}

Global comparisons between peak wall stress (PWS) predicted for each patient (from FE) and maximum and peak USPIO uptake $(\% \Delta \mathrm{T} 2 *)$ per patient were also investigated, using values derived from the entire aneurysm. Maximum AAA diameter was also included in the analysis, as this is the most widely used predictor of aneurysm rupture. To test if correlations varied with classification of USPIO uptake (i.e. USPIO-negative or USPIOpositive), comparisons by group were also investigated.

\section{Mural USPIO Enhancement}

To examine the trends with respect to the focal mural inflammation observed in USPIO-positive aneurysms more closely, the correlation of diameter and whole-vessel PWS with mural USPIO uptake values (mean and peak USPIO values identified on the most diseased segment) was investigated. Nonfocal areas of USPIO uptake (i.e. those which did not meet the definition of mural USPIO enhancement) were removed prior to this analysis.

\section{Statistical Analysis}

Linear dependence between variables was investigated using the Pearson's correlation coefficient. Unpaired twosample $t$ tests were used to assess differences in mean values between AAA groups. Statistical significance was determined using a two-tailed $p<0.05$. All statistical analyses were performed using Minitab ${ }^{\circledR} 17$ (Minitab Ltd., Coventry CV 2TE, UK).

\section{Results}

Fifty patients were included in this study who were predominantly elderly (mean age of $72(65-87)$ years) men $(90 \%)$, with a mean CT AAA diameter of $52.96(40.60-69.40) \mathrm{mm}$. Mural USPIO enhancement was seen in $21(42 \%)$ patients who had a significantly greater mean AAA diameter (55.19 vs $51.34 \mathrm{~mm}$ without mural enhancement, $p=0.0255$ ). There was no difference between groups in terms of other relevant characteristics (Table 1). 
Table 1 Summary demographics of patients, per USPIO classification group, using comparisons of proportion or chi-squared test, where appropriate

\begin{tabular}{|c|c|c|c|c|}
\hline Variable & $\begin{array}{l}\text { All patients } \\
(n=50)\end{array}$ & $\begin{array}{l}\text { USPIO-negative } \\
(n=29)\end{array}$ & $\begin{array}{l}\text { USPIO-positive } \\
(n=21)\end{array}$ & $\begin{array}{l}\text { Difference between } \\
\text { groups }-p \text { value }\end{array}$ \\
\hline Age in years (SD) & $72.34(6.32)$ & $71.96(6.37)$ & $72.86(6.37)$ & 0.6372 \\
\hline Male sex $(\%)$ & $45(90)$ & $25(86)$ & $20(95)$ & 0.383 \\
\hline Mean AAA diameter in mm (SD) & $52.96(6.08)$ & $51.34(5.19)$ & $55.19(6.62)$ & 0.0255 \\
\hline \multicolumn{5}{|l|}{ Past medical history } \\
\hline Family history of AAA (\%) & $12(24)$ & $6(20.69)$ & $6(28.57)$ & 0.738 \\
\hline Coronary artery disease $(\%)$ & $16(32)$ & $9(31.03)$ & $7(33.33)$ & 1 \\
\hline Stroke or transient ischemic attack $(\%)$ & $3(6)$ & $2(6.90)$ & $1(4.76)$ & 1 \\
\hline Peripheral vascular disease $(\%)$ & $3(6)$ & $3(10.34)$ & $0(0)$ & 0.254 \\
\hline Cerebrovascular disease $(\%)$ & $2(4)$ & $2(6.90)$ & $0(0)$ & 0.503 \\
\hline \multicolumn{5}{|l|}{ Risk factors } \\
\hline Current smoking habit (\%) & $12(24)$ & $5(17.24)$ & $7(33.33)$ & 0.314 \\
\hline Previous smoking habit (\%) & $32(64)$ & $20(68.97)$ & $12(57.14)$ & 0.551 \\
\hline Hypertension $(\%)$ & $40(80)$ & $24(82.76)$ & $16(76.20)$ & 0.7232 \\
\hline Hypercholesterolaemia (\%) & $44(88)$ & $25(86.21)$ & $19(90.48)$ & 1 \\
\hline Diabetes $(\%)$ & $7(14)$ & $4(13.79)$ & $3(14.29)$ & 1 \\
\hline \multicolumn{5}{|l|}{ Medication } \\
\hline Anti-diabetes medication (\%) & $6(12)$ & $5(17.24)$ & $1(4.76)$ & 0.38 \\
\hline Statin therapy $(\%)$ & $2(4)$ & $2(6.90)$ & $0(0)$ & 0.503 \\
\hline Anti-coagulant therapy (\%) & $1(2)$ & $0(0)$ & $1(4.76)$ & 0.42 \\
\hline
\end{tabular}

\section{D Contour Plot and USPIO Colour Map Comparison}

The maximum areas of PWS were most commonly $(n=28$; $56 \%$ ) found in the posterior wall and regions of high curvature such as the shoulder region. When analysing the most diseased segment, 40 aneurysms (80\%) demonstrated some degree of visual overlap between regions of elevated stress and USPIO enhancement anywhere (e.g. adjacent to lumen and/or wall) in the vessel, while in the remaining 10 cases, no visual overlap was observed (e.g. Fig. 2b). Of the 40 aneurysms which did exhibit overlap, only 19 (38\%) demonstrated spatial co-location of increased stress and USPIO enhancement adjacent to the aneurysm wall, while the remaining aneurysms exhibited overlap of elevated stress at areas of peri-luminal USPIO uptake where classification is challenging (e.g. Fig. $2 c)$. Overall, only eight aneurysms (16\%) demonstrated colocation of elevated stress with an area meeting the definition of mural USPIO enhancement (e.g. Fig. 2d).

Details of inter- and intra-observer variability for stress and USPIO comparisons as well as additional contour plot comparisons for the full 50 patients can be found in the supplementary text (ESM 1).

\section{Global Comparisons: Whole AAA Analysis}

The average PWS for all aneurysms was $0.1980 \mathrm{MPa}$. There was no difference between the average PWS for USPIOnegative aneurysms $(0.1999 \pm 0.1326 \mathrm{MPa})$ and USPIO- positive aneurysms $(0.1955 \pm 0.1495 \mathrm{MPa} ; p=0.83,95 \%$ CI -0.0920 to 0.0740 ).

Maximum diameter was not associated with PWS $(r=0.13, p=0.36)$ or maximum USPIO uptake $(r=0.05$, $p=0.74)$. There was no correlation between PWS and maximum USPIO enhancement over the entire aneurysm $(r=0.17$, $p=0.23$ ), as shown in Fig. 3 .

When comparing between groups, diameter was not correlated with PWS in either group (USPIO-negative $r=0.17$, $p=0.39$; USPIO-positive $r=0.13, p=0.61$ ), nor was diameter and peak USPIO uptake (USPIO-negative $r=0.04, p=0.85$; USPIO-positive $r=-0.15, p=0.52$ ), as shown in Fig. 4. There was no difference in correlation between PWS and mural USPIO enhancement between groups $(r=0.23$ vs. $r=0.09$; $p=0.22$ ).

\section{Focal Mural USPIO Enhancement}

There were no associations between PWS and mean or peak USPIO uptake within the individual areas of mural USPIO enhancement on the most diseased segment $(r=0.37, p=0.10$; $r=0.32, p=0.16$ ), as shown in Fig. 5a and b respectively.

Similarly, mean USPIO uptake within the identified regions of mural USPIO enhancement did not correlate with diameter $(r=-0.05, p=0.61)$, as shown in Fig. 6a. However, a significant weak inverse correlation was observed between peak USPIO uptake and diameter $(r=-0.45$, $p=0.04)$, as shown in Fig. 6b. 

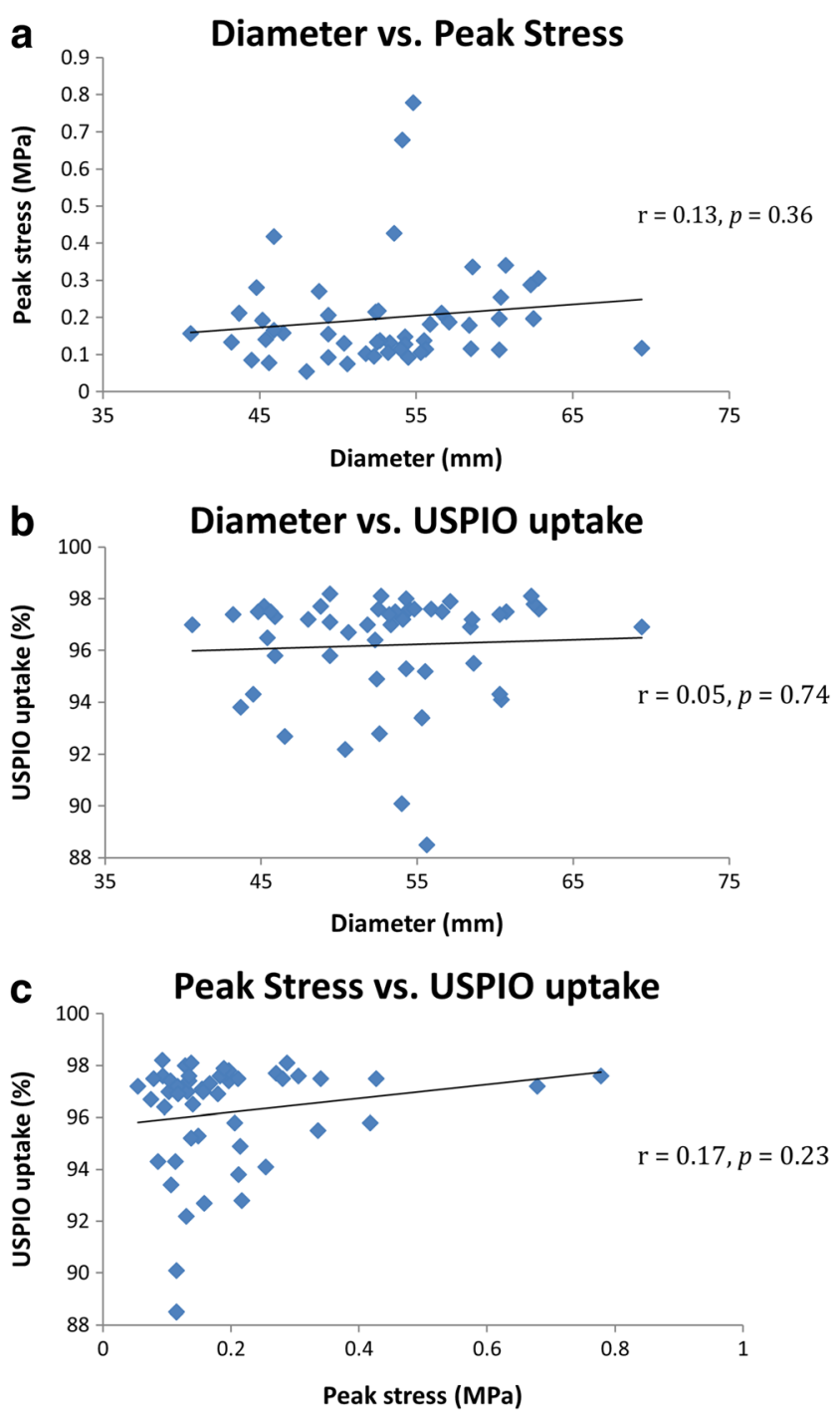

Fig. 3 Global comparisons $(n=50)$ of aneurysm diameter, peak stress predicted by finite element analysis, and $\% \Delta \mathrm{T} 2 * \mathrm{USPIO}$, using data from the entire aneurysm. There are no significant correlations between any of the measured parameters

\section{Discussion}

In the present study, we analysed the spatial relationship between two biomarkers of AAA rupture risk; namely, inflammation detected through the uptake of USPIO and wall stress calculated using patient-specific FE models. Elevated stress was commonly observed in areas vulnerable to rupture (e.g. the posterior wall and shoulder), while regions of USPIO enhancement typically occurred near the wall behind thick thrombus. Only $16 \%$ of aneurysms exhibited co-localisation of elevated stress and mural USPIO enhancement. Globally, no correlation was observed between peak stress and other measures of USPIO uptake, e.g. mean or peak uptake.

The maximal area of PWS (as demonstrated using FE modelling) is most commonly found in the posterior aneurysm wall, where thrombus is typically thin or absent and the blood pool is in close proximity. Up to $82 \%$ of AAA ruptures occur on the posterior wall [27], yet most aneurysms bulge anteriorly. This may be explained by the restriction in radial expansion of the posterior wall due to the spinal column, with increasing anterior asymmetry resulting in an increase in posterior wall peak stress [28]. In addition, PWS was also frequently observed in regions of high curvature, including the shoulder region. These findings have been reported in many previous FE studies [29, 30], some of which have demonstrated that increased stress at the aneurysm shoulder is associated with AAA expansion [11], possibly due to stress-induced changes in vessel wall stiffness [3].

Despite there being general visual overlap between stress and USPIO uptake, only $16 \%$ of all aneurysms in our study demonstrated co-location of elevated stress with mural USPIO enhancement. The finding of co-location of elevated stress and USPIO uptake in the posterior wall is challenging because this is a very thin structure and we cannot resolve whether this represents mural or periluminal uptake of USPIOs. Periluminal USPIO uptake is non-specific and reflects the passive trapping of USPIOs within the gelatinous thrombus immediately adjacent to the lumen. Histological analysis of aneurysm tissue has demonstrated that the immediate luminal aspect consists of freshly deposited thrombus that is highly cellular (including macrophages) with progressive organisation towards the abluminal surface, which is acellular and consists largely of fibrin [31]. Therefore, the presence of USPIO uptake in the fibrinous thrombus adjacent to the aneurysm wall can be considered to represent true inflammation, whereas periluminal uptake of USPIO does not represent true cellular inflammation. Gadolinium-based MRI studies appear to reiterate this - the luminal surface of thrombus enhances with gadolinium, in contrast to the organised part of the thrombus and the aortic wall, which typically do not [8].

With regards to co-location of PWS and mean, maximum, or peak USPIO uptake, no significant associations were demonstrated. These findings suggest that areas of elevated stress and objective measures of focal inflammation (as demonstrated on USPIO-enhanced MRI) do not commonly co-locate in AAA disease. While we know that focal mechanical and biological processes do contribute towards AAA disease progression and rupture, it appears that stress and inflammation represent two distinct processes that are not necessarily connected spatially or causally. Our previous pilot study of USPIO MRI demonstrated a link between regions of focal cellular inflammation and future AAA expansion. Indeed, histological analysis of tissue obtained during AAA repair provided evidence of the accumulation of USPIO within macrophages, and USPIO-positive aneurysms were found to expand three times more rapidly than USPIO-negative aneurysms [8].

Other molecular imaging studies of AAA wall inflammation using positron emission tomography (PET) have demonstrated the ability of ${ }^{18} \mathrm{~F}$-FDG to identify regions of inflammation, confirmed by histological analysis [32]. However, 
Fig. 4 Comparisons of diameter, peak stress, and \% $\Delta \mathrm{T} 2 *$ USPIO uptake per group; USPIOnegative $(n=29)$ vs USPIOpositive $(n=21)$, using data from the entire aneurysm. No significant correlations demonstrated
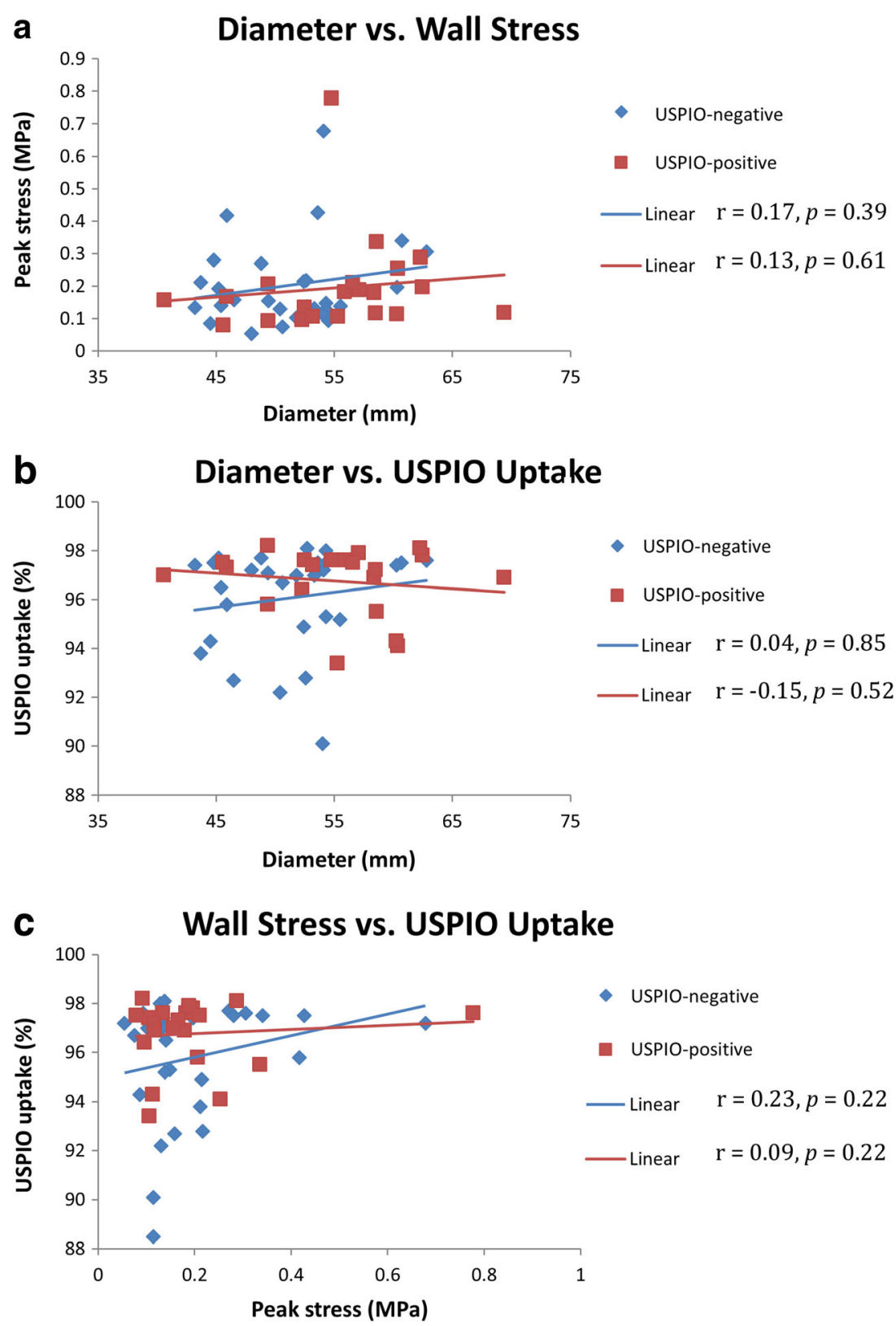

there is no definite consensus as to whether increased ${ }^{18} \mathrm{~F}$ FDG uptake correlates with clinical outcomes such as expansion or rupture $[33,34]$. In addition, we recently examined vascular inflammation in a small population of AAAs $(n=15)$ and found some but not a close correlation between ${ }^{18}$ F-FDG uptake on PET/CT and USPIO uptake on MRI [17]. This may reflect the different elements of macrophage activity that these imaging techniques detect: glycolysis and phagocytosis, respectively.

Some other studies have also attempted to quantify the interaction between inflammation and peak stress, using a combined mechanical and biological approach to assess the overall stability of individual AAAs. In a pilot study $(n=5)$, $\mathrm{Xu}$ et al. reported a tentative link between ${ }^{18} \mathrm{~F}$-FDG metabolic activity and high wall stress [30]. Later work by Maier et al. confirmed that wall stress predicted by FE and ${ }^{18}$ F-FDG uptake evaluated by PET/CT correlated both quantitatively and spatially in the cases examined $(n=18)$ [29]. Interestingly, our study has observed that high stress often occurs in regions of high curvature or inflection points, such as the aneurysm shoulder and posterior luminal surface, which have been reported to have high ${ }^{18}$ F-FDG uptake [6, 17, 29, 30]. However, more recent work by Nchimi and colleagues [6] on a larger sample size $(n=53)$ concluded that the relationship between ${ }^{18}$ F-FDG uptake and peak stress was not directly correlated, instead pointing to a complex multi-factorial relationship between increased ${ }^{18} \mathrm{~F}$-FDG uptake and patient-specific factors such as aneurysm location (thoracic or abdominal), wall 

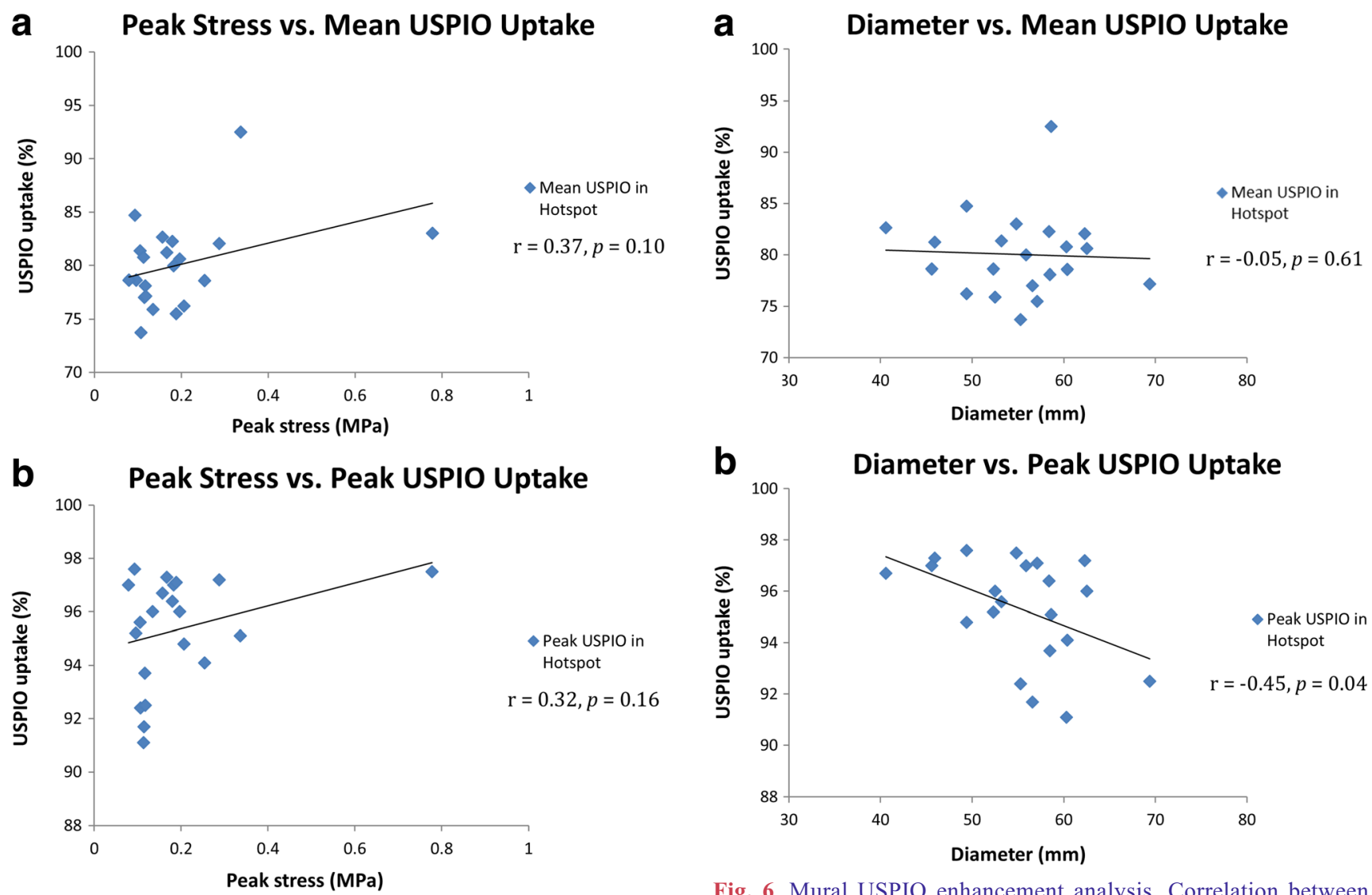

Fig. 5 Mural USPIO enhancement analysis. Correlation between peak stress (derived from the entire aneurysm) with mean and peak USPIO uptake on the most diseased segment $(n=21)$. No significant correlations identified

stress, and family history/patient lifestyle. The present study also suggests that the relationship between inflammation and peak stress is complex and potentially independent.

\section{Translational Impact and Clinical Implications}

While each marker (e.g. USPIO uptake or stress) provides an independent indicator of aneurysm rupture risk, neither presents the full picture in isolation. However, when a combined approach such as presented in this study is taken, then added insight into the mechanical and biological conditions of the wall can be provided where indicators from these two independent markers coincide. Such insights may potentially lead to new methods for patient risk stratification, in particular in patients below the standard $55 \mathrm{~mm}$ intervention threshold or in larger aneurysms where the benefit/risk of intervention is less certain.

\section{Limitations and Future Direction}

Our study has some technical limitations. We employed a continuum modelling strategy for the intraluminal thrombus, which

Fig. 6 Mural USPIO enhancement analysis. Correlation between aneurysm diameter with mean and peak USPIO uptake in the identified areas of significant mural USPIO enhancement on the most diseased segment $(n=21)$

is thought to act as a mechanical buffer [35], meaning that stress behind thick thrombus may be under-represented. Improvements in thrombus modelling may improve this [36]; however, our strategy is in keeping with most previous studies. In addition, our regions of mural USPIO enhancement were identified on axial slices in the 2D plane - this is based on the techniques used in our previous pilot study and is considered a reasonable, pragmatic approach to image analysis. However, our group is also exploring 3D mural USPIO enhancement detection [37], which may provide additional data with which to compare wall stress in the future. Furthermore, as in many previous studies, we have assumed population-mean parameters for the AAA material properties, which may influence the resulting wall stress. Ongoing work aims to eliminate the potential uncertainty surrounding material properties [38], and it is hoped that this step in combination with a more comprehensive comparison of USPIO-uptake and PWS in 3D may provide some further insight into the complex interplay of these two factors in AAA disease progression. One final limitation is the lack of longitudinal data with which to verify our hypothesis that overlapping regions of stress and USPIO may potentially identify patients at greater risk of rupture. Recent findings from the main $\mathrm{MA}^{3} \mathrm{RS}$ study [39] have already demonstrated that 
USPIO uptake predicts expansion, but that this is not independent of diameter. Final follow-up data from the trial has been collected, and we have recently started to explore other secondary analyses, including how baseline stress/USPIO uptake predictions relate to the evolution of the aneurysm over time. However, given the non-trivial nature of this work, it will take many months before the data is fully analysed and interpreted. Once analysed, it is anticipated that these findings will provide a greater level of insight into disease progression and the significance of the overlapping regions of stress and inflammation.

\section{Conclusions}

In this combined clinical and FE study of 50 aneurysms, poor correlations between USPIO uptake and stress suggest that these biological and mechanical factors address different aspects of the aberrant pathway towards disease progression in AAA. We observed that peak stress most commonly occurs in regions of increased curvature (such as the posterior wall and inflection points), and further correlation with peak stress and different markers of inflammation is warranted. While both macrophage-mediated inflammation and peak wall stress play a part in AAA expansion and rupture, they do not spatially colocate, and this only serves to reinforce the complex multifactorial elements in aneurysm disease progression. However, it remains a possibility that although PWS and mural USPIO enhancement are independent processes, when they co-localise, this could be a trigger for aneurysm rupture. To address this hypothesis requires long-term follow-up of clinical cohorts. Additional clinical and biomechanical studies are therefore required to further investigate the synergy between biological and biomechanical aspects of AAA disease.

Acknowledgements The authors would like to gratefully acknowledge the staff at the Clinical Research Imaging Centre (CRIC), University of Edinburgh, for facilitating access to the imaging data.

\section{Compliance with Ethical Standards}

Funding Funding for this work was provided through the National Institute of Health Research (NIHR) Efficacy and Mechanism Evaluation (EME) Programme as part of the $\mathrm{MA}^{3} \mathrm{RS}$ clinical trial (NIHR EME 11/20/03).

Conflict of Interest One of the authors (CW) was in part supported by funding from the Toshiba Medical Visualization Systems (Europe) Ltd.

Ethical Approval The study was approved by a local research ethics committee (East of Scotland Ethics Research Service, ref 12/ES/0068) and performed in accordance with the Declaration of Helsinki.

Informed Consent Informed written consent was obtained for all patients prior to participation in the study.
Open Access This article is distributed under the terms of the Creative Commons Attribution 4.0 International License (http:// creativecommons.org/licenses/by/4.0/), which permits unrestricted use, distribution, and reproduction in any medium, provided you give appropriate credit to the original author(s) and the source, provide a link to the Creative Commons license, and indicate if changes were made.

\section{References}

1. Mitchell, D., Hindley, H., Naylor, R., Wyatt, M., \& Loftus, I. (2012). The abdominal aortic aneurysm quality improvement programme. The Vascular Society of Great Britain \& Ireland.

2. Choke, E., Cockerill, G., Wilson, W. R., Sayed, S., Dawson, J., \& Loftus, I. (2005). A review of biological factors implicated in abdominal aortic aneurysm rupture. European Journal of Vascular and Endovascular Surgery, 30(3), 227-244. doi: 10.1016/j.ejvs.2005.03. 009 .

3. Helderman, F., Manoch, I. J., Breeuwer, M., Kose, U., Schouten, O., Sambeek, M. R. M., et al. (2008). A numerical model to predict abdominal aortic aneurysm expansion based on local wall stress and stiffness. Medical \& Biological Engineering \& Computing, 46(11), 1121-1127. doi:10.1007/s11517-008-0358-3.

4. O’Leary, S. A., Mulvihill, J. J., Barrett, H. E., Kavanagh, E. G., Walsh, M. T., McGloughlin, T. M., et al. (2015). Determining the influence of calcification on the failure properties of abdominal aortic aneurysm (AAA) tissue. Journal of the Mechanical Behavior of Biomedical Materials, 42, 154-167. doi:10.1016/j.jmbbm.2014.11.005.

5. Humphrey, J. D., \& Holzapfel, G. A. (2012). Mechanics, mechanobiology, and modeling of human abdominal aorta and aneurysms. Journal of Biomechanics, 45(5), 805-814. doi:10. 1016/j.jbiomech.2011.11.021.

6. Nchimi, A., Cheramy-Bien, J. P., Gasser, T. C., Namur, G., Gomez, P., \& Seidel, L. (2014). Multifactorial relationship between ${ }^{18} \mathrm{~F}$ fluoro-deoxy-glucose positron emission tomography signaling and biomechanical properties in unruptured aortic aneurysms. Circulation: Cardiovascular Imaging, 7(1), 82-91. doi: 10.1161/ circimaging.112.000415 .

7. Forsythe, R. O., Newby, D. E., \& Robson, J. M. J. (2016). Monitoring the biological activity of abdominal aortic aneurysms beyond ultrasound. Heart. doi:10.1136/heartjnl-2015-308779.

8. Richards, J. M., Semple, S. I., MacGillivray, T. J., Gray, C., Langrish, J. P., Williams, M., et al. (2011). Abdominal aortic aneurysm growth predicted by uptake of ultrasmall superparamagnetic particles of iron oxide: a pilot study. Circulation. Cardiovascular Imaging, 4(3), 274-281. doi:10.1161/circimaging.110.959866.

9. Sadat, U., Taviani, V., Patterson, A. J., Young, V. E., Graves, M. J., Teng, Z., et al. (2011). Ultrasmall superparamagnetic iron oxide-enhanced magnetic resonance imaging of abdominal aortic aneurysms - a feasibility study. European Journal of Vascular and Endovascular Surgery, 41(2), 167-174. doi:10. 1016/j.ejvs.2010.08.022.

10. Malkawi, A. H., Hinchliffe, R. J., Xu, Y., Holt, P. J., Loftus, I. M., \& Thompson, M. M. (2010). Patient-specific biomechanical profiling in abdominal aortic aneurysm development and rupture. Journal of Vascular Surgery, 52(2), 480-488. doi:10.1016/j.jvs.2010.01.029.

11. Li, Z.-Y., Sadat, U., U-King-Im, J., Tang, T. Y., Bowden, D. J., Hayes, P. D., et al. (2010). Association between aneurysm shoulder stress and abdominal aortic aneurysm expansion: a longitudinal follow-up study. Circulation, 122(18), 1815-1822. doi:10.1161/ circulationaha.110.939819.

12. Conlisk, N., Geers, A. J., McBride, O. M. B., Newby, D. E., \& Hoskins, P. R. (2016). Patient-specific modelling of abdominal aortic aneurysms: the influence of wall thickness on predicted clinical 
outcomes. Medical Engineering \& Physics, 38(6), 526-537. doi:10. 1016/j.medengphy.2016.03.003.

13. Xenos, M., Rambhia, S. H., Alemu, Y., Einav, S., Labropoulos, N., Tassiopoulos, A., et al. (2010). Patient-based abdominal aortic aneurysm rupture risk prediction with fluid structure interaction modeling. [journal article]. Annals of Biomedical Engineering, 38(11), 3323-3337. doi:10.1007/s10439-010-0094-3.

14. Doyle, B. J., McGloughlin, T. M., Miller, K., Powell, J. T., \& Norman, P. E. (2014). Regions of high wall stress can predict the future location of rupture of abdominal aortic aneurysm. [journal article]. Cardiovascular and Interventional Radiology, 37(3), 815818. doi:10.1007/s00270-014-0864-7.

15. Vallabhaneni, S. R., Gilling-Smith, G. L., How, T. V., Carter, S. D., Brennan, J. A., \& Harris, P. L. (2004). Heterogeneity of tensile strength and matrix metalloproteinase activity in the wall of abdominal aortic aneurysms. Journal of Endovascular Therapy, 11(4), 494-502. doi:10.1583/04-1239.1.

16. McBride, O. M. B., Berry, C., Burns, P., Chalmers, R. T. A., Doyle, B., Forsythe, R., et al. (2015). MRI using ultrasmall superparamagnetic particles of iron oxide in patients under surveillance for abdominal aortic aneurysms to predict rupture or surgical repair: MRI for abdominal aortic aneurysms to predict rupture or surgery - the MA3RS study. Open Heart, 2(1), e000190. doi:10. 1136/openhrt-2014-000190.

17. McBride, O. M. B., Joshi, N. V., Robson, J. M. J., MacGillivray, T. J., Gray, C. D., Fletcher, A. M., et al. (2016). Positron emission tomography and magnetic resonance imaging of cellular inflammation in patients with abdominal aortic aneurysms. European Journal of Vascular and Endovascular Surgery, 51(4), 518-526. doi:10.1016/j.ejvs.2015.12.018.

18. Raghavan, M. L., Vorp, D. A., Federle, M. P., Makaroun, M. S., \& Webster, M. W. (2000). Wall stress distribution on threedimensionally reconstructed models of human abdominal aortic aneurysm. Journal of Vascular Surgery, 31(4), 760-769. doi:10. 1067/mva.2000.103971.

19. Gasser, T. C., Auer, M., Labruto, F., Swedenborg, J., \& Roy, J. (2010). Biomechanical rupture risk assessment of abdominal aortic aneurysms: model complexity versus predictability of finite element simulations. European Journal of Vascular and Endovascular Surgery, 40(2), 176-185. doi:10.1016/j.ejvs.2010.04.003.

20. Martufi, G., Satriano, A., Moore, R. D., Vorp, D. A., \& Di Martino, E. S. (2015). Local quantification of wall thickness and intraluminal thrombus offer insight into the mechanical properties of the aneurysmal aorta. [journal article]. Annals of Biomedical Engineering, 43(8), 1759-1771. doi:10.1007/s10439-014-1222-2.

21. Shang, E. K., Nathan, D. P., Woo, E. Y., Fairman, R. M., Wang, G. J., Gorman, R. C., et al. (2013). Local wall thickness in finite element models improves prediction of abdominal aortic aneurysm growth. Journal of Vascular Surgery. doi:10.1016/j.jvs.2013.08.032.

22. Shum, J., DiMartino, E. S., Goldhamme, A., Goldman, D. H., Acker, L. C., Patel, G., et al. (2010). Semiautomatic vessel wall detection and quantification of wall thickness in computed tomography images of human abdominal aortic aneurysms. Medical Physics, 37(2), 638-648.

23. Raghavan, M. L., \& Vorp, D. A. (2000). Toward a biomechanical tool to evaluate rupture potential of abdominal aortic aneurysm: identification of a finite strain constitutive model and evaluation of its applicability. Journal of Biomechanics, 33(4), 475-482. doi: 10.1016/S0021-9290(99)00201-8.

24. Wang, D. H. J., Makaroun, M., Webster, M. W., \& Vorp, D. A. (2001). Mechanical properties and microstructure of intraluminal thrombus from abdominal aortic aneurysm. [article]. Journal of Biomechanical Engineering, 123(6), 536-539. doi:10.1115/1.1411971.

25. Doyle, B. J., Callanan, A., Walsh, M., Grace, P., \& McGloughlin, T. (2009). A finite element analysis rupture index (FEARI) as an additional tool for abdominal aortic aneurysm rupture prediction. Vascular Disease Prevention, 6, 114-121.
26. Vorp, D. A., Raghavan, M. L., \& Webster, M. W. (1998). Mechanical wall stress in abdominal aortic aneurysm: influence of diameter and asymmetry. Journal of Vascular Surgery, 27(4), 632-639. doi:10.1016/S0741-5214(98)70227-7.

27. Fillinger, M. F., Racusin, J., Baker, R. K., Cronenwett, J. L., Teutelink, A., \& Schermerhorn, M. L. (2004). Anatomic characteristics of ruptured abdominal aortic aneurysm on conventional CT scans: implications for rupture risk. Journal of Vascular Surgery, 39(6), 1243-1252. doi: 10.1016/j.jvs.2004.02.025

28. Doyle, B. J., Callanan, A., Burke, P. E., Grace, P. A., Walsh, M. T., Vorp, D. A., et al. (2009). Vessel asymmetry as an additional diagnostic tool in the assessment of abdominal aortic aneurysms. Journal of Vascular Surgery, 49(2), 443-454. doi:10.1016/j.jvs.2008.08.064.

29. Maier, A., Essler, M., Gee, M. W., Eckstein, H.-H., Wall, W. A., \& Reeps, C. (2012). Correlation of biomechanics to tissue reaction in aortic aneurysms assessed by finite elements and $\left[{ }^{18} \mathrm{~F}\right]-$ fluorodeoxyglucose-PET/CT. Int. J. Numer. Method. Biomed. Eng., 28(4), 456-471. doi:10.1002/cnm.1477.

30. Xu, X. Y., Borghi, A., Nchimi, A., Leung, J., Gomez, P., \& Cheng, Z. (2010). High levels of ${ }^{18} \mathrm{~F}$-FDG uptake in aortic aneurysm wall are associated with high wall stress. European Journal of Vascular and Endovascular Surgery, 39(3), 295-301. doi: 10.1016/j.ejvs.2009.10. 016 .

31. Gasser, T. C., Martufi, G., Auer, M., Folkesson, M., \& Swedenborg, J. (2010). Micromechanical characterization of intra-luminal thrombus tissue from abdominal aortic aneurysms. [journal article]. Annals of Biomedical Engineering, 38(2), 371-379. doi:10.1007/ s10439-009-9837-4.

32. Courtois, A., Nusgens, B. V., Hustinx, R., Namur, G., Gomez, P., \& Somja, J. (2013). ${ }^{18}$ F-FDG uptake assessed by PET/CT in abdominal aortic aneurysms is associated with cellular and molecular alterations prefacing wall deterioration and rupture. Journal of Nuclear Medicine, 54(10), 1740-1747. doi: 10.2967/jnumed.112.115873.

33. Kotze, C. W., Groves, A. M., Menezes, L. J., Harvey, R., Endozo, R., \& Kayani, I. A. (2011). What is the relationship between (1)(8)F-FDG aortic aneurysm uptake on PET/CT and future growth rate? European Journal of Nuclear Medicine and Molecular Imaging, 38, 1493-1499. doi:10.1007/s00259-011-1799-8.

34. Reeps, C., Essler, M., Pelisek, J., Seidl, S., Eckstein, H. H., \& Krause, B. J. (2008). Increased ${ }^{18} \mathrm{~F}$-fluorodeoxyglucose uptake in abdominal aortic aneurysms in positron emission/computed tomography is associated with inflammation, aortic wall instability, and acute symptoms. Journal of Vascular Surgery, 48(2), 417-423. doi: 10.1016/j.jvs.2008.03.059

35. Doyle, B., \& McGloughlin, T. (2011). Computer-aided diagnosis of abdominal aortic aneurysms. In T. McGloughlin (Ed.), Biomechanics and mechanobiology of aneurysms (vol. 7, studies in mechanobiology, tissue engineering and biomaterials) (pp. 119-138). Berlin: Springer.

36. Ayyalasomayajula, A., Vande Geest, J. P., \& Simon, B. R. (2010). Porohyperelastic finite element modeling of abdominal aortic aneurysms. Journal of Biomechanical Engineering, 132(10), 104502. doi:10.1115/1.4002370.

37. Koutraki, Y. G., Forsythe, R. O., McBride, O. M. B., Wang, C., Robson, J. M. J., MacGillivray, T. J., et al. (2016). Automatic classification and 3D visualisation of abdominal aortic aneurysms to predict aneurysm expansion and rupture. In Paper presented at the ISMRM 24th Annual Meeting \& Exhibition, Singapore, 07-13 May 2016.

38. Joldes, G. R., Miller, K., Wittek, A., \& Doyle, B. (2016). A simple, effective and clinically applicable method to compute abdominal aortic aneurysm wall stress. Journal of the Mechanical Behavior of Biomedical Materials, 58, 139-148. doi:10.1016/j.jmbbm.2015.07.029.

39. Study Investigators, T. M. R. (2017). Aortic wall inflammation predicts abdominal aortic aneurysm expansion, rupture and need for surgical repair. Circulation. doi:10.1161/circulationaha.117.028433. 